\title{
Biocompatible ionic liquid enhances transdermal antigen peptide delivery and preventive vaccination effect
}

Yoshiro Tahara, ${ }^{1, \dagger,+}$ Kaho Morita, ${ }^{1, \dagger}$ Rie Wakabayashi, ${ }^{1,3}$ Noriho Kamiya, ${ }^{1,2,3}$ and Masahiro Goto ${ }^{1,2,3, *}$

${ }^{1}$ Department of Applied Chemistry, Graduate School of Engineering, ${ }^{2}$ Center for Future Chemistry, and ${ }^{3}$ Center for Advanced Transdermal Drug Delivery System Center, Kyushu University, 744 Motooka, Nishi-ku, Fukuoka 819-0395, Japan

*Corresponding author, e-mail: m-goto@mail.cstm.kyushu-u.ac.jp.

†These authors contributed equally.

Fresent address: Faculty of Science and Engineering, Doshisha University, 1-3 Tatara Miyakodani,

Kyotanabe City, Kyoto 610-0321, Japan 


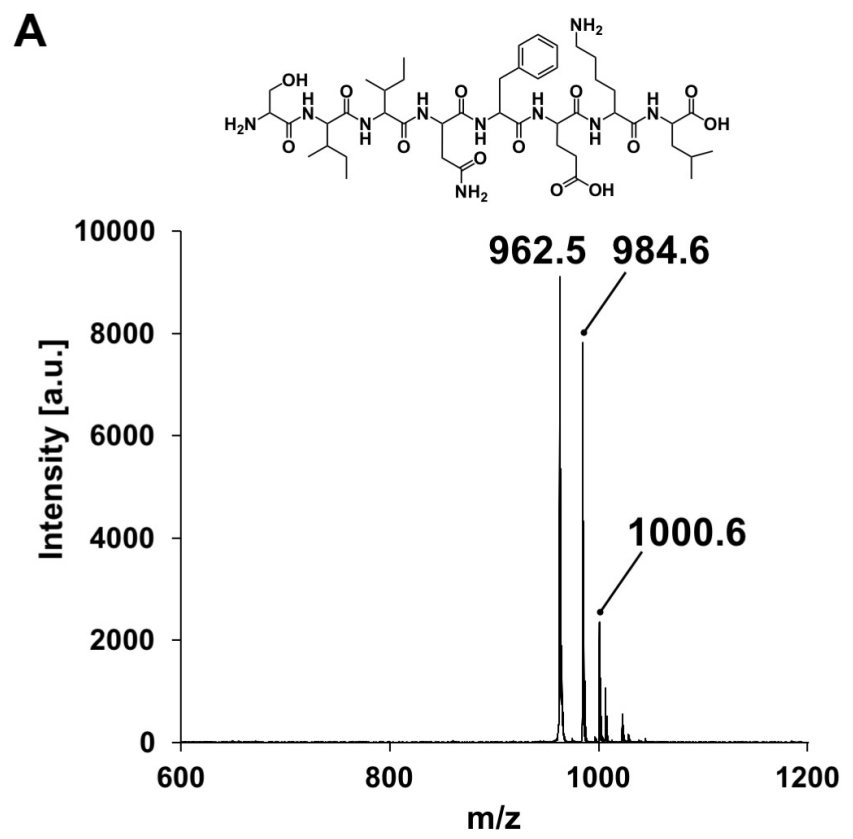

B
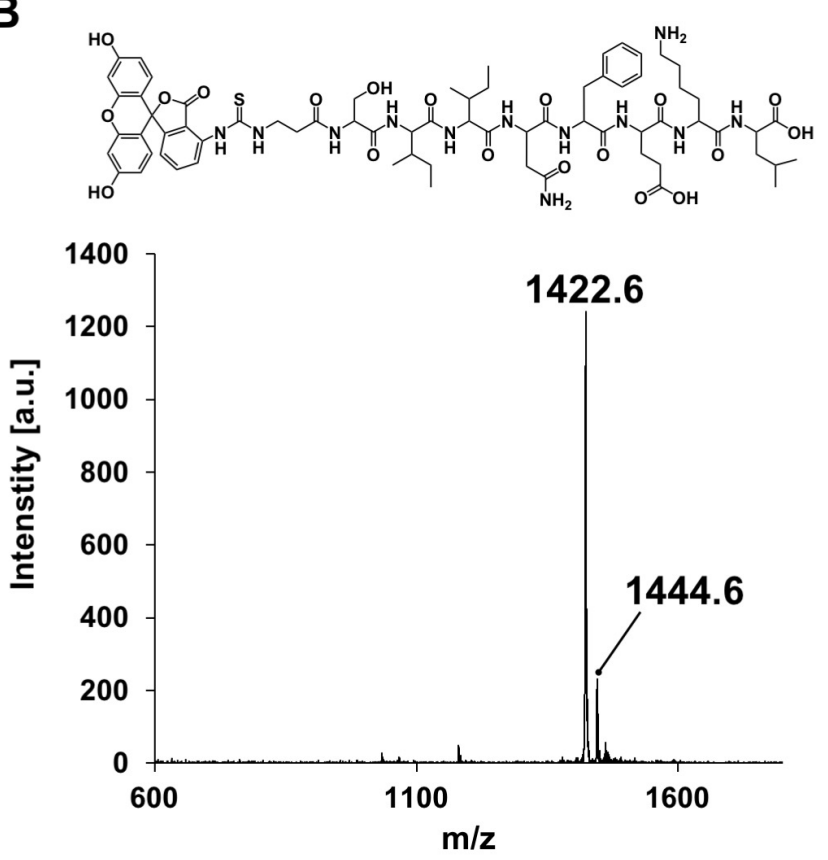

Figure S1. Chemical structure and the result of MALDI-TOF-MS of (A) peptide (SIINFEKL) and (B) FITC-peptide (FITC- $\beta$-Ala-SIINFEKL). 


\section{A [Cho][C8]}

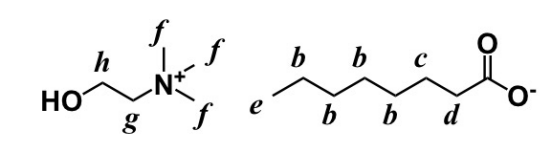

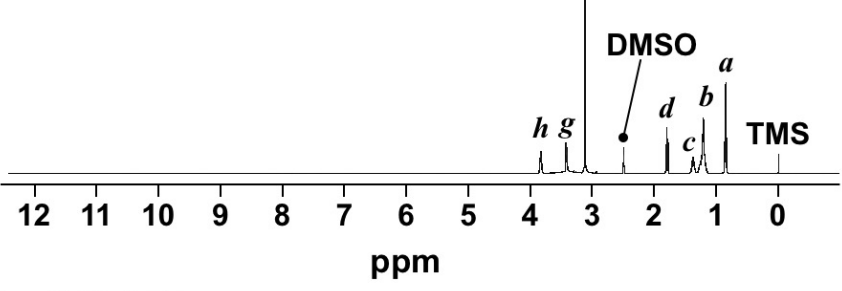

\section{B [Cho][C10]}

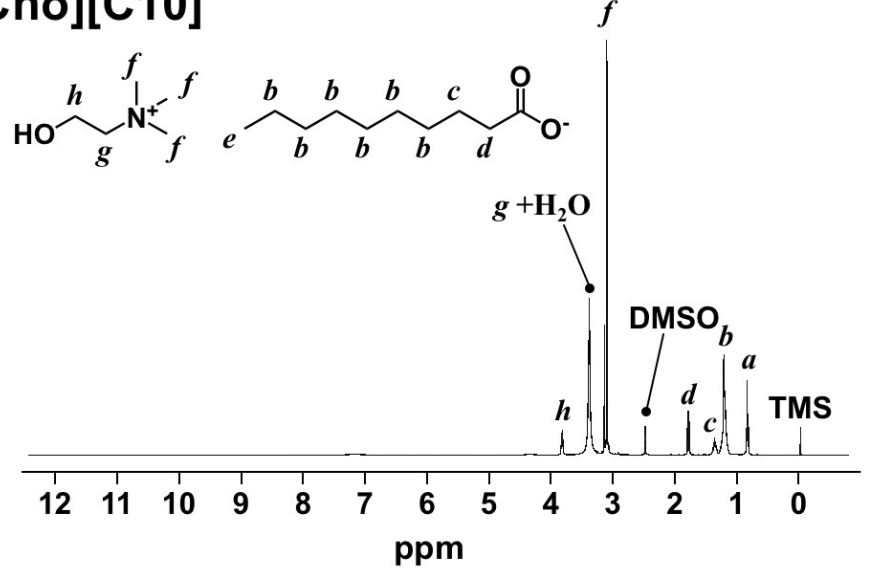

\section{C [Cho][C12]}

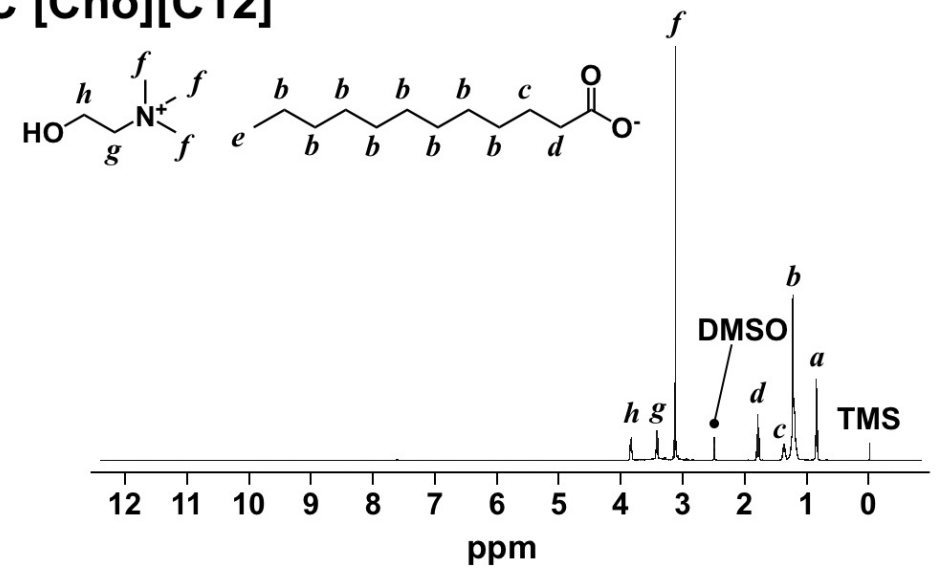

\section{D [Cho][C14]}

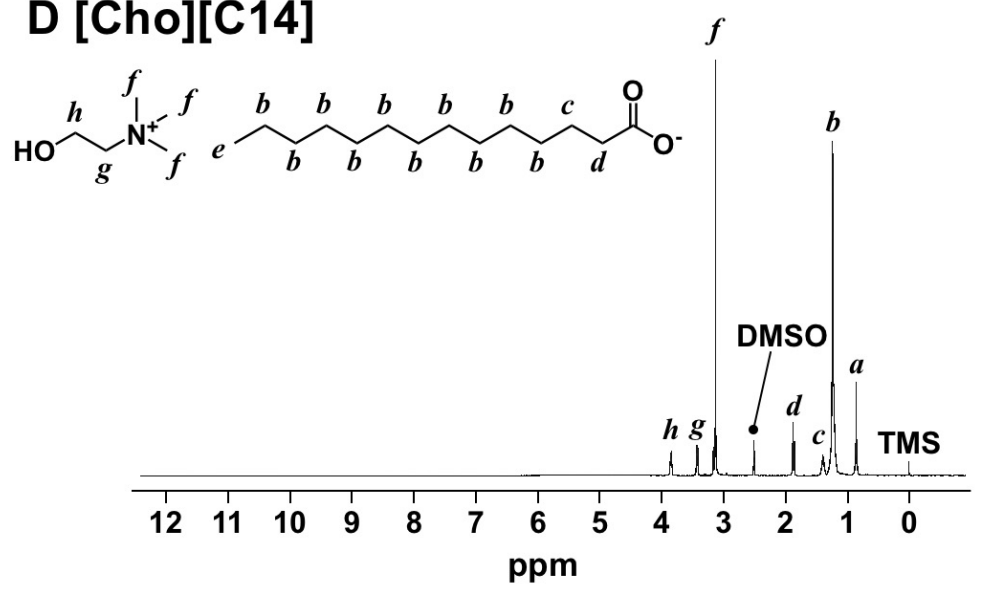

Figure S2. (Continued) 


\section{$E[C h o][C 16]$}

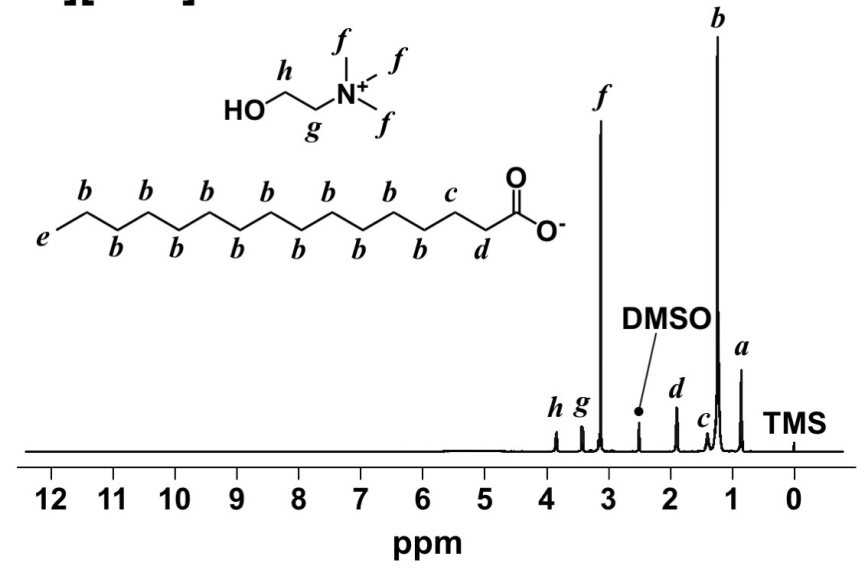

F [Cho][C18:0]

HO $\overbrace{g}^{h} \underbrace{f}_{f} N_{f}^{f} f$

$\overbrace{b}^{b} b \overbrace{b}^{b} \underbrace{b}_{b} \underbrace{b}_{b} \underbrace{b}_{b} \underbrace{c}_{d} \|_{0}$

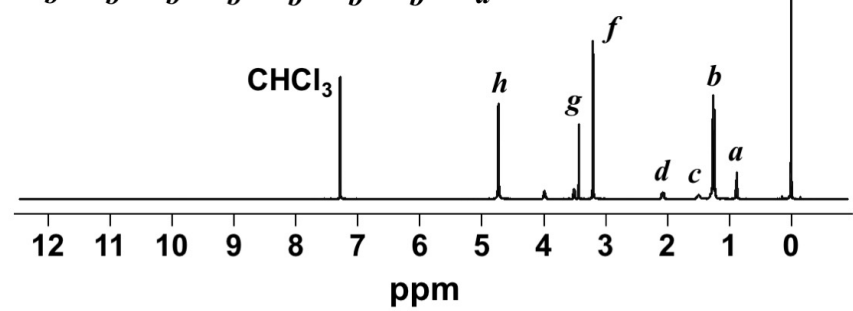

\section{G [Cho][C18:1]}

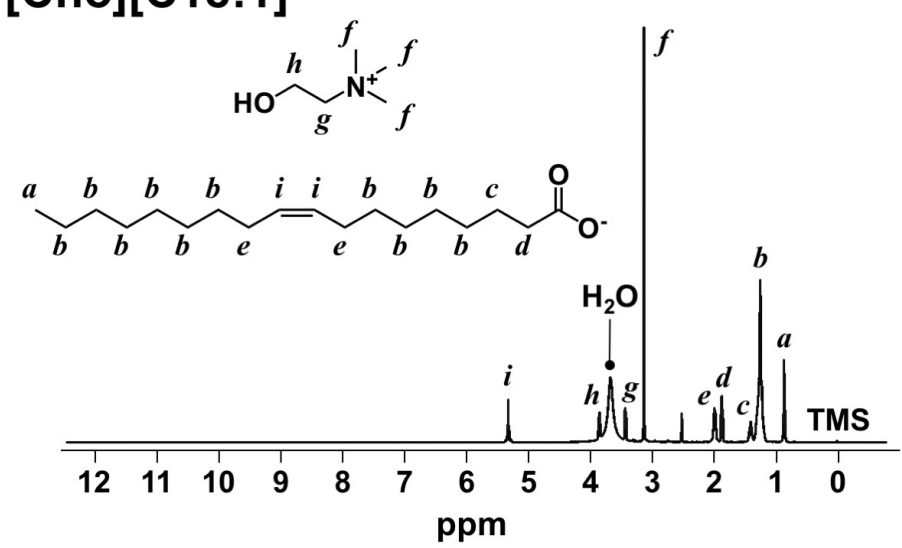

Figure S2. ${ }^{1} \mathrm{H}-\mathrm{NMR}$ spectrum of (A) [Cho][C8] in $\mathrm{d}_{6}-\mathrm{DMSO}$, (B) [Cho][C10] in $\mathrm{d}_{6}-\mathrm{DMSO},(\mathrm{C})$ [Cho][C12] in $\mathrm{d}_{6}$-DMSO, (D) [Cho][C14] in $\mathrm{d}_{6}-\mathrm{DMSO}$, (E) [Cho][C16] in $\mathrm{d}_{6}-\mathrm{DMSO}$, (F) [Cho][C18:0] in $\mathrm{CDCl}_{3}$ and $(\mathrm{G})[\mathrm{Cho}][\mathrm{C} 18: 1]$ in $\mathrm{d}_{6}$-DMSO. 


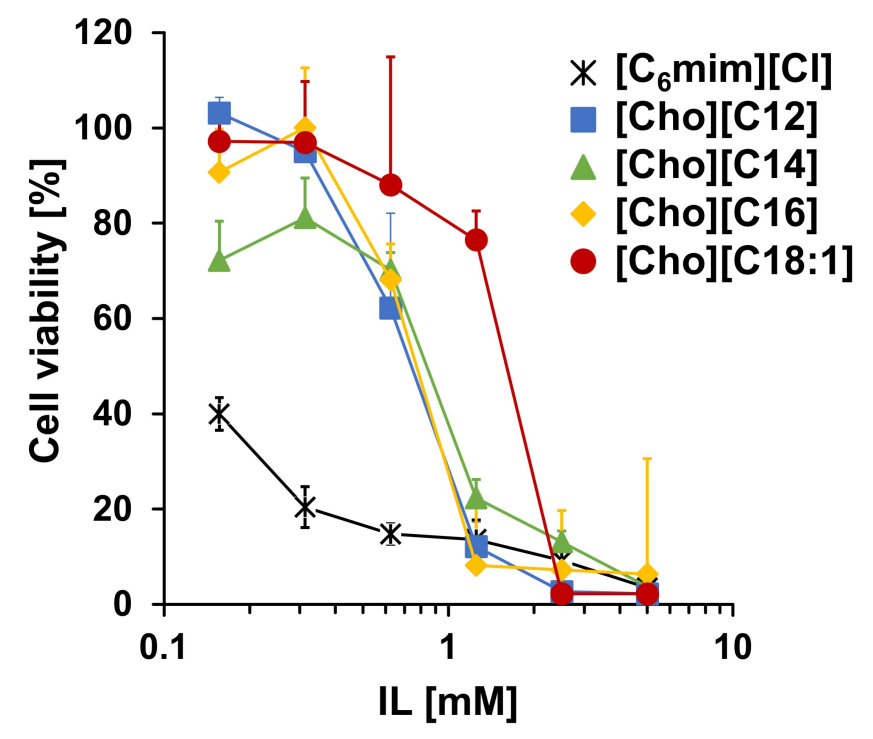

Figure S3. Cell viability of DC2.4 cells treated with [Cho][FA] and control samples for $24-\mathrm{h} . \quad N=3$.
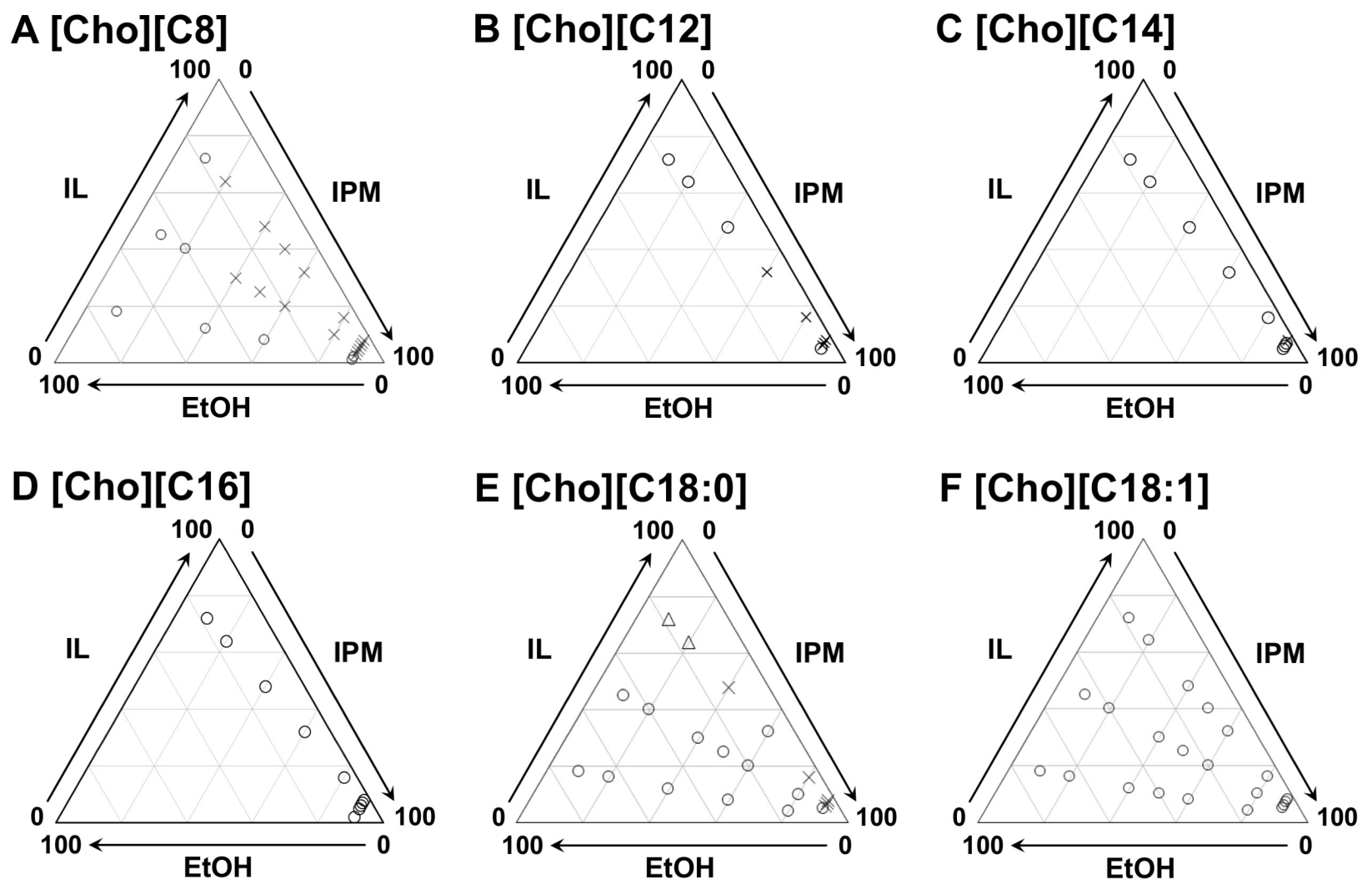

\section{$\bigcirc$ : Soluble $\quad \Delta$ : Suspension $\quad x$ : Precipitation}

Figure S4. Pseudoternary phase diagram of IL, EtOH and IPM. 

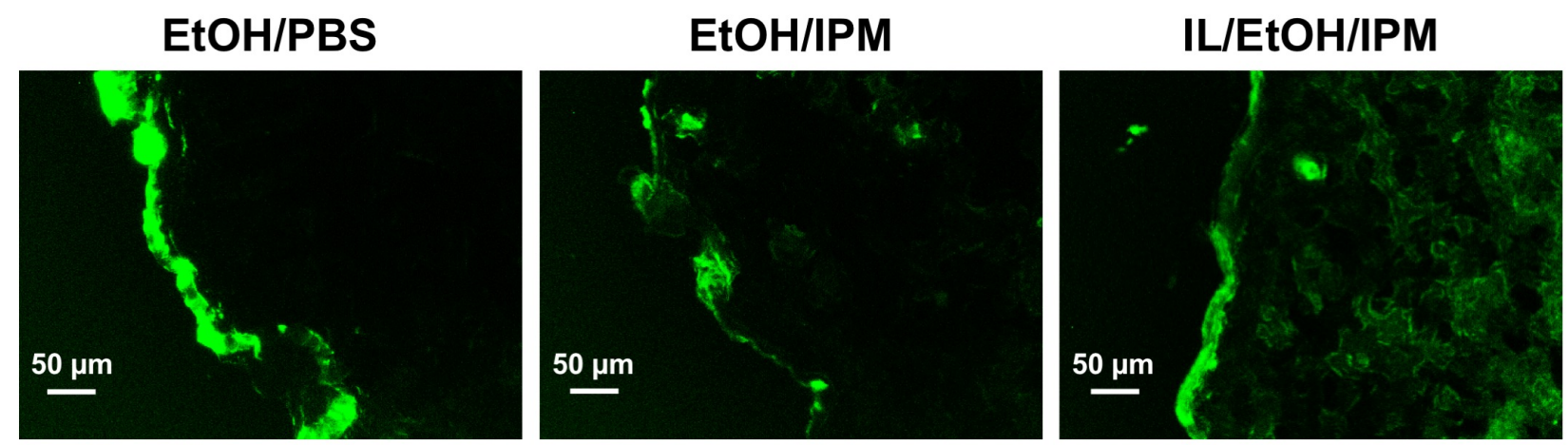

Figure S5. Cross section of mouse skin after $4 \mathrm{~h}$ treatment of FITC-peptide in EtOH/PBS, EtOH/IPM and IL/EtOH/IPM.

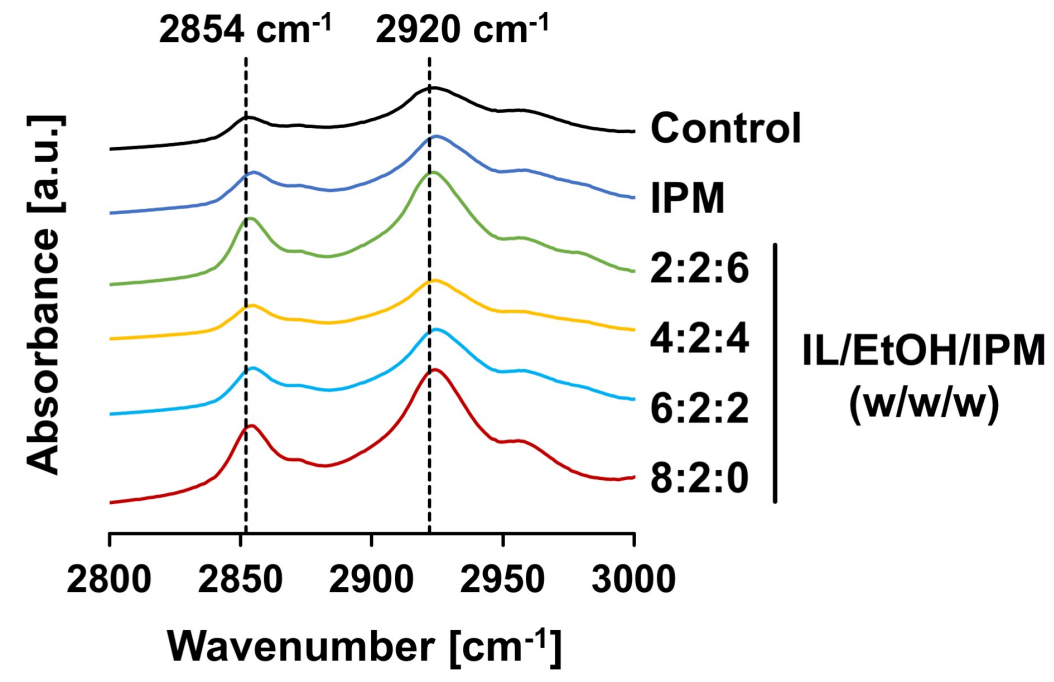

Figure S6. FITR spectra of the lipid region for SC. 


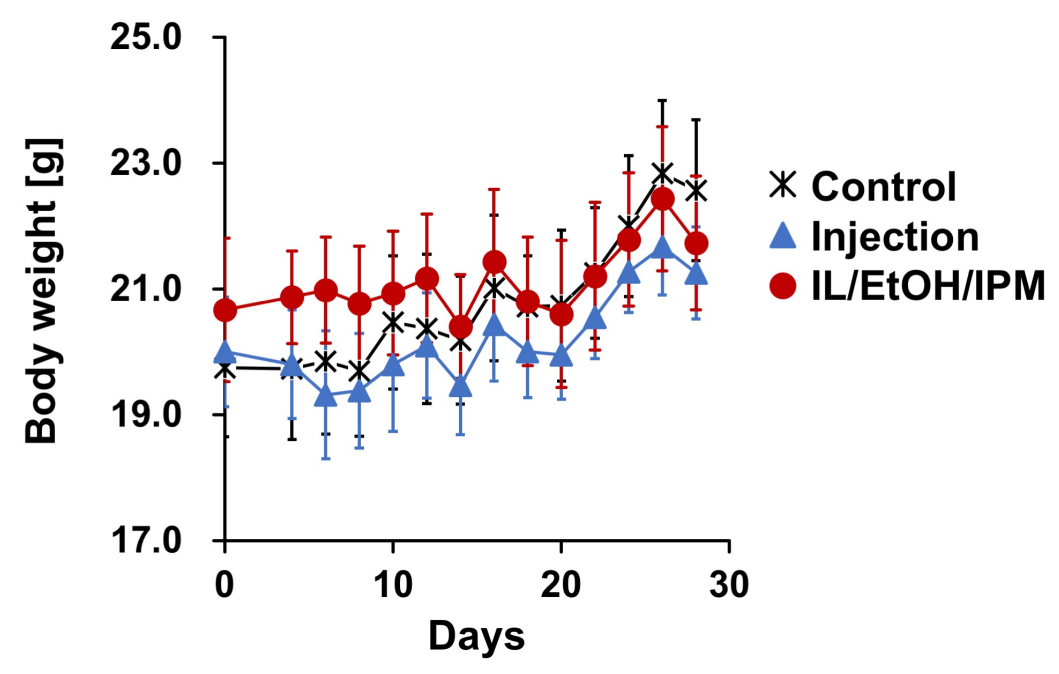

Figure S7. Body weight of mice from one week after twice immunization at one week interval. Samples and tumor inoculation are same as Figure 7. $N=6$.

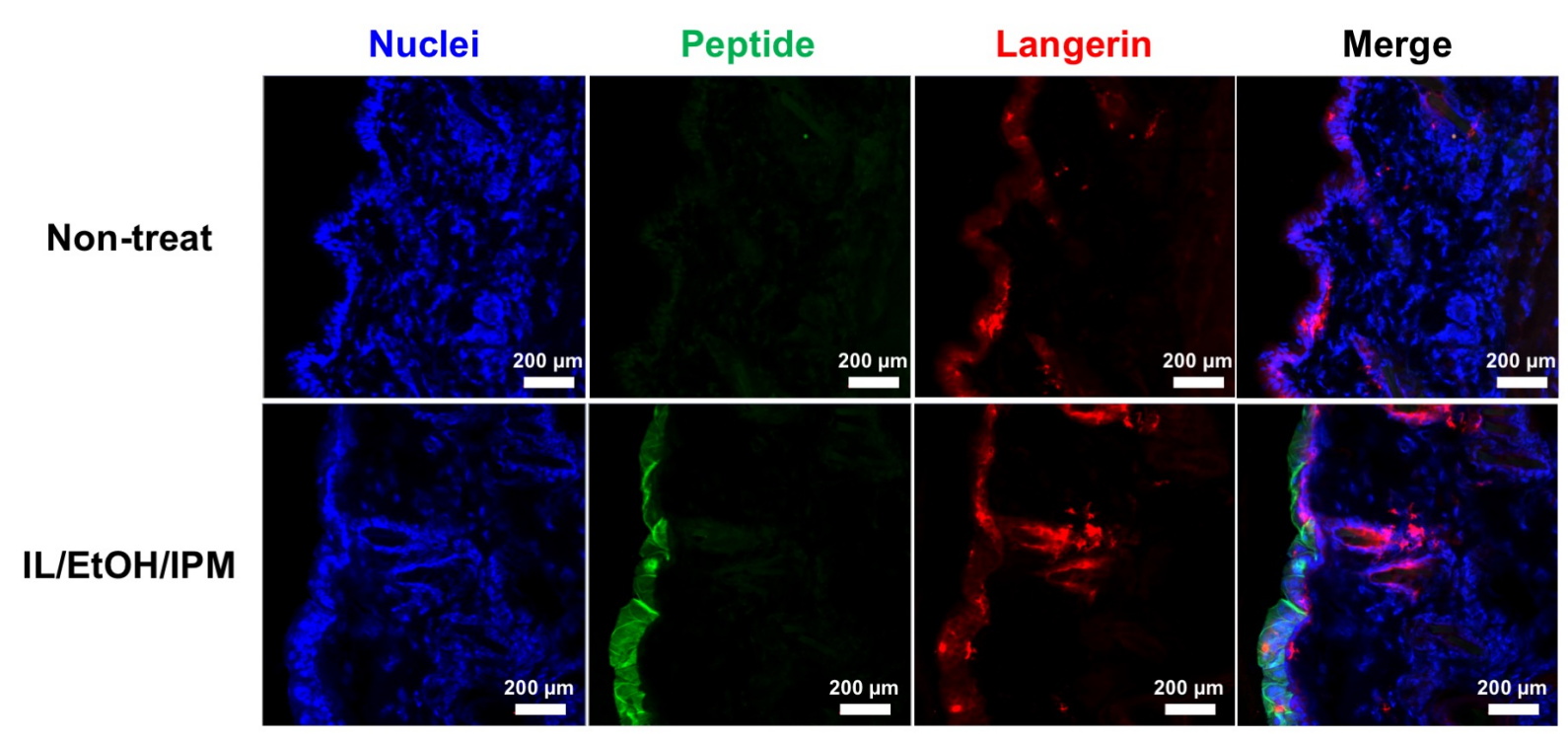

Figure S8. Immunehistochemical evaluation of peptide uptake by langerin-positive antigen presenting cells in the skin. Blue: DAPI (nuclei). Green: FITC-peptide. Red: PE-labeled anti-langerin (CD207) antibody. 
Table S1 Elemental Analysis of [Cho][C18:1]

\begin{tabular}{llll}
\hline [Cho][C18:1] & C & H & N \\
\hline Found & $69.68 \%$ & $11.96 \%$ & $3.37 \%$ \\
Calc. for [Cho][C18:1]- $\mathrm{H}_{2} \mathrm{O}$ & $68.44 \%$ & $12.24 \%$ & $3.47 \%$ \\
\hline
\end{tabular}

Table S2 Melting points of [Cho][FA]. ${ }^{a}$ ND: not detectable due to its low stability. ${ }^{b}$ Ref: G. Knothe and R.O. Dunn, J. Am. Oil Chem. Soc., 86, 843-856 (2009).

\begin{tabular}{lcll}
\hline ILs & Melting point $\left[{ }^{\circ} \mathbf{C}\right]$ & Fatty acids & Melting point $\left[{ }^{\circ} \mathbf{C}\right]^{b}$ \\
\hline$[$ Cho $][\mathrm{C} 8]$ & $\mathrm{ND}^{a}$ & Caprylic acid (C8) & 16.5 \\
{$[\mathrm{Cho}][\mathrm{C} 10]$} & 10 & Capric acid (C10) & 31.5 \\
{$[\mathrm{Cho}][\mathrm{C} 12]$} & 22 & Lauric acid (C12) & 44 \\
{$[\mathrm{Cho}][\mathrm{C} 14]$} & 28 & Myristic acid (C14) & 58 \\
{$[\mathrm{Cho}][\mathrm{C} 16]$} & 43 & Palmitic acid (C16) & 63 \\
{$[\mathrm{Cho}][\mathrm{C} 18: 0]$} & 56 & Stearic acid (C18:0) & 71.2 \\
{$[$ Cho $][\mathrm{C} 18: 1]$} & -27 & Oleic acid (C18:1) & 16.3 \\
\hline
\end{tabular}

Table S3 Skin permeation parameters calculated from the result of Figure 5A $(N=3) \cdot{ }^{a}$ Parameters of EtOH/PBS were calculated from one sample because the two data of three samples of EtOH/PBS were low and hard to calculate. NS: not significant, ${ }^{* * *} p<0.001$, compared with EtOH/IPM.

\begin{tabular}{llll}
\hline & EtOH/PBS & EtOH/IPM & IL/EtOH/IPM \\
\hline Flux $J\left(\mu \mathrm{g} \mathrm{cm}^{-2} \mathrm{~h}^{-1} \times 10^{-2}\right)$ & $1.03^{a}$ & $3.19 \pm 1.68$ & $28.7 \pm 2.9^{* * *}$ \\
Lag time $t_{\text {lag }}(\mathrm{h})$ & $2.9^{a}$ & $3.01 \pm 0.32$ & $2.54 \pm 0.11^{\mathrm{NS}}$ \\
Diffusion coefficient $D\left(\mathrm{~cm}^{2} \mathrm{~h}^{-1} \times 10^{-5}\right)$ & $9.54^{a}$ & $9.35 \pm 0.29$ & $11.1 \pm 0.5^{\mathrm{NS}}$ \\
Partition coefficient $K(-)$ & $133^{a}$ & $136 \pm 64$ & $1062 \pm 73^{* * *}$ \\
\hline
\end{tabular}

